\title{
Taxanes Versus Vinorelbine and 5-Fluorourocil as a first Line Therapy in Metastatic Breast Cancer.
}

\author{
Ibtessam Saad El-Din', Wael Samir'1, Wessam El-Sherief', Yomna Khaiery². \\ ${ }^{1}$ Kasr El-Aini Oncology Center (NEMROCK)., ${ }^{2}$ El-Salam Oncology Center
}

Purpose: To compare the efficacy and tolerance of taxanes versus a combination of vinorelbine and 5-FU in the treatment of MBC previously treated with anthracyclines and analyzing the effect of various prognostic factors.

Material and methods: Between April 2005 and April 2007, sixty eligible MBC patients were randomized between 2 arms. Arm I received taxanes (either paclitaxel $175 \mathrm{mg} / \mathrm{m}^{2}$ D1 or Docetaxel $100 \mathrm{mg} / \mathrm{m} 2 \mathrm{D} 1$ ) and arm II received combination of vinorelbine $25 \mathrm{mg} / \mathrm{m} 2 \mathrm{D} 1$, D5 and $5-\mathrm{FU} 600 \mathrm{mg} / \mathrm{m}^{2}$ by continuous IV infusion D1 to D5. cycles of chemotherapy were repeated every 21 to 28 days for a total of 6 cycles in both arms.

Results: Three patients (10.3\%) in arm I achieved complete remission versus one patient (3.4\%) in arm II, while 13 patients $(44.8 \%)$ in arm I versus 9 patients $(31 \%)$ in arm II had partial remission. $(P=0.383)$. Neurosensory changes and fluid retention were more evident in arm I while diarrhea and nail changes were more common in arm II $(P=0.03)$. The mean overall survival was 27.7 months with taxanes and 26.5 months with vinorelbine and 5-FU $(P=0.79)$ while progression free survival was 15.7 months in patients treated in arm I versus 8.5 months in arm II $(P=0.234)$. Conclusion: Both regimens are well tolerated and feasible, there treatment outcome and toxicity profile are comparable but cost effective issue may have an important impact on treatment selection.

Key words: Metastatic breast cancer, taxanes, vinorelbine and 5-FU.

Corresponding Author: Wael Samir

E-mail: wael_makar@yahoo.com

\section{INTRODUCTION}

Breast cancer continuous to be the most common cancer in women world-wide. Metastatic breast cancer is usually considered as an incurable situation for which treatment chosen to control the disease should take into account the maintenance of a good quality of life. The clinical benefit which encompasses objective response and long standing stability of the disease has often become a goal in metastatic setting ${ }^{1}$. Also, improvement in time to progression and duration of response have been considered as primary goals of treatment ${ }^{2}$.

Eventually, chemotherapy is considered for almost all patients with metastatic breast cancer. Several agents are now available offering expanded treatment options for many patients ${ }^{3}$.

Combination regimens should be considered for patients with overwhelming symptoms or rapidly progressive or life threatening metastasis for which a combination regimen is more likely to result in a tumor response ${ }^{4}$.

Response rates to initial therapy with anthracyclines, taxanes, capecitabine, vinorelbine and gemecitaline range on average from $25 \%$ to $60 \%$ with median time to progression averaging approximately 6 months ${ }^{4}$.

The aim of this study is to compare the efficacy and tolerance of taxanes versus combination of intravenous vinorelbine and 5-FU given by continuous infusion in the treatment of metastatic breast cancer patients previously treated with anthracyclines and analyzing the effect of various prognostic factors in both regimens.

\section{PATIENTS AND METHODS}

This study is a randomized prospective study that included 60 patients with metastatic breast cancer presenting to Kasr El-Aini Oncology Center (NEMROCK) and El-Salam Oncology Center in the period from April 2005 to April 2007.

\section{Patient eligibility criteria:}

1. Patients age range from 18 to 70 years.

2. WHO performance status of 0 to 2 .

3. Pathologically proved breast cancer with evidence of visceral, bone or locoregional disease.

4. All patients should have received been treated with an anthracycline based chemotherapy in the adjuvant setting. 


Vol. 7| No. 3-4 $2011 \quad$ Wessam Ali El-Sherief, et al.

Patients were randomized between two arms. Arm I received taxanes (either paclitaxel or docetaxel) and arm II received vinorelbine and 5-Flurouracil.

\section{Study design and treatment:}

Thirty patients were treated in arm I by a single agent, taxanes either docetaxal $100 \mathrm{mg} / \mathrm{m}^{2}$ D1 1 hour intravenous infusion or paclitxel $175 \mathrm{mg} / \mathrm{m}^{2} \mathrm{D} 13$ hours intravenous infusion, to be repeated after 3 to 4 weeks. Patients were given antiemetics, antihistaminecs and corticosteroids as premedications before receiving the taxane and continued two days after.

Arm II included also 30 patients treated by a combination chemotherapy protocol consisting of vinorelbine (Navelbine) given intravenously in a dose of $25 \mathrm{mg} / \mathrm{m}^{2}$ D1 and D5 together with 5-Fluorouracil $600 \mathrm{mg} / \mathrm{m} 2$ by a continuous infusion from D1 to D5 of the cycle, cycles to be repeated 21 to 28 days provided clinical and hematological recovery.

Actual body weight was used to calculate body surface area and after an amendment to the study protocol the maximum body surface was limited to $2.0 \mathrm{~m}^{2}$. Clinical, hematological and biochemical assessments were required before each cycle, including assessment of toxic effects according to the common toxicity criteria, version 1 of the National Cancer Institute.

In both arms patients were re-evaluated by physical examination, radiological imaging and laboratory tests after completion of 3 cycles of the chemotherapy protocol to assess the response as well as the efficacy and toxicity of the treatment.

Patients showing disease progression were planned to shift to another line of chemotherapy while those showing response or stable disease continued on the same line of treatment to a total of 6 cycles in both treatment arms.

\section{Statistical Analysis:}

Data were statistically described in terms of range, mean, standard deviation $( \pm \mathrm{SD})$, median, frequencies (number of cases) and relative frequencies (percentages) where appropriate. Comparison of age between the study groups was done using Mann Whitney T-test for independent samples for comparing categorical data, Chi square (x2) test was; performed. Yates correction was used instead when the frequency is less than 10. Survival, analysis was done for the different outcome measures using Kaplan Mayer statistics with the corresponding survival graphs, A probability value ( $P$-value) less than 0.05 was considered, statistically significant. All statistical calculations were done using computer programs. Microsoft Excel version seven (Microsoft
Corporation, NY, USA) and SPSS (Statistical Package for the Social Science; SPSS Inc., Chicago, IL, USA) statistical program.

\section{RESULTS}

The characteristics of the 60 patients enrolled in the study are summarized in (Table 1).

\section{Symptoms:}

A variety of presenting symptoms were found according to the different metastatic sites.

The cardinal symptom was pain either in the right hypochondrium related to the liver metastasis of patients $9 / 30(30 \%)$ in arm I and 5/30 (16.7\%) in arm II (P- 0.22) or in bony metastatic sites $8 / 30(26.7 \%)$ in arm I and $12 / 30(40 \%)$ in arm II $(P=0.206)$.

Chest symptoms (dyspnea, cough+expectoration) were found in $36.7 \%(11 / 30)$ of patients in arm I and $(30 \%) 9 / 30$ in arm II $(P=0.392)$

Swelling (in the breast or lymph nodes) is $3 / 30$ $(10 \%)$ of patients in arm I and 4/30 (13.3\%) in arm II ( $p$-value 0.5$)$. Skin nodules in $12 / 30(40 \%)$ of patients in arm I and 11/30(36.7) in arm II $(P=0.792)$.

Number of metastatic sites in both arms are summarized in (Table 2).

\section{Treatment related toxicity:}

Treatment related toxicities were measured from grade $0-4$ according to the WHO recommendations as regards hematological toxicities (Table 3 ) and according to the EORTC classification and recommendation for grading of non-hematological toxicities (Table 4).

\section{Response:}

The assessment of response was carried out at the end of the treatment course.

Patients who achieved Complete Response (CR) were $3 / 30(10.3 \%)$ in arm I (received taxanes), versus $1 / 30(3.4 \%)$ in arm II (received navelbine and 5-FU).

Thirteen patients (44.8\%) in arm I had Partial Remission (PR) versus 9 patients (31\%) in arm II.

While patients who developed disease progression were 3 patients $(10.3 \%)$ in arm I versus 6 patients (31\%) in arm II.

Patients who had Stationary Disease (SD) were $11 / 30(36.6 \%)$ in arm I versus 14/30 (46\%). 
Kasr-El-Aini Journal Of Clinical Oncology And Nuclear Medicine

The results of the response rate were statistically non significant between the two arms $(P=0.383)$.

\section{Survival:}

The mean overall survival (OAS) was 27.7 months in arm I versus 26.5 months in arm II $(P=0.79)$ (Fig. 1), while the Progression Free Survival (PFS) for arm I was 15.7 months and 8.5 months for arm I $(P=0.234)$ (Fig. 2).

\section{Factors affecting response rate:}

Using univariant analysis different prognostic factors were studied in relation to response, time to progression and overall survival.

Factors as age ( $>35$ years), site of recurrence (local or bone) and number of metastatic sites $(<2$ sites $)$ show statistically significant difference (Table 5).

Other factors as performance status (PS), Menopausal Status (MS) and hormonal status (HS) show no statistically significant difference (Table 6).

Table 1: Patients characteristics.

\begin{tabular}{|c|c|c|c|c|c|c|}
\hline \multirow{2}{*}{ Parameter } & & \multicolumn{2}{|c|}{ Arm } & \multicolumn{2}{|c|}{ Arm II } & \multirow{2}{*}{$P$-value } \\
\hline & & Number & Percent & Number & Percent & \\
\hline \multirow[t]{2}{*}{ Age in years } & $<35$ & 5 & $16.5 \%$ & 7 & $23.3 \%$ & \\
\hline & $>35$ & 25 & $83 \%$ & 23 & $76.6 \%$ & 0.112 \\
\hline \multirow[t]{3}{*}{ Performance status } & 0 & 5 & $17.2 \%$ & 4 & $14.8 \%$ & \\
\hline & 1 & 18 & $62.1 \%$ & 19 & $66.7 \%$ & \\
\hline & 2 & 6 & $20.7 \%$ & 5 & $18.5 \%$ & 0.581 \\
\hline \multirow[t]{2}{*}{ Menopausal status } & Pre & 10 & $33.3 \%$ & 11 & $37.9 \%$ & \\
\hline & Post & 20 & $66.7 \%$ & 18 & $62.1 \%$ & 0.789 \\
\hline \multirow[t]{2}{*}{ Pathology } & IDC & 26 & $86.6 \%$ & 24 & $80 \%$ & \\
\hline & ILC & 4 & $13.3 \%$ & 6 & $19 \%$ & 0.551 \\
\hline \multirow[t]{2}{*}{ Tumor grade } & II & 20 & $66.7 \%$ & 22 & $73.3 \%$ & \\
\hline & III & 10 & $33.3 \%$ & 8 & $26.7 \%$ & 0.576 \\
\hline \multirow[t]{2}{*}{ ER group } & $+\mathrm{ve}$ & 18 & $60.0 \%$ & 22 & $73.3 \%$ & \\
\hline & -ve & 12 & $40.0 \%$ & 8 & $26.7 \%$ & 0.412 \\
\hline \multirow[t]{2}{*}{ PR group } & $+\mathrm{ve}$ & 17 & $56.6 \%$ & 18 & $60 \%$ & \\
\hline & -ve & 13 & $43.4 \%$ & 12 & $40 \%$ & 0.562 \\
\hline \multirow[t]{2}{*}{ Her 2neu } & $+\mathrm{ve}$ & 3 & $10 \%$ & 0 & $0 \%$ & \\
\hline & -ve & 5 & $16.6 \%$ & 3 & $10 \%$ & 0.339 \\
\hline \multirow[t]{4}{*}{ Site of metastasis } & Visceral: Liver & 12 & $40 \%$ & 8 & $26.7 \%$ & 0.206 \\
\hline & Lung & 12 & $40 \%$ & 9 & $30 \%$ & 0.294 \\
\hline & Loco & 15 & $50 \%$ & 14 & $46.7 \%$ & 0.500 \\
\hline & Bone & 9 & $30 \%$ & 12 & $40 \%$ & 0.294 \\
\hline
\end{tabular}

Table 2: Number of metastatic sites in both arms.

\begin{tabular}{lccccc}
\hline & \multicolumn{2}{c}{ Arm I } & \multicolumn{2}{c}{ Arm II } & \multirow{2}{*}{$\boldsymbol{P}$-value } \\
\cline { 1 - 4 } Number of metastatic sites & No & $\%$ & No & $\%$ & \\
\cline { 1 - 4 } One site & 15 & 50 & 16 & 53.3 & \\
\cline { 1 - 4 } Two sites & 12 & 40 & 14 & 46.7 & 0.386 \\
\cline { 1 - 4 } Three sites or more & 3 & 10 & 0 & 0 &
\end{tabular}

Table 3: Hematological toxicities.

\begin{tabular}{lcccccc}
\hline & & \multicolumn{2}{c}{ Arm I } & \multicolumn{2}{c}{ Arm II } & \multirow{2}{*}{-value } \\
\cline { 1 - 6 } Parameter & & No & $\%$ & No & $\%$ & \\
\hline Neutropenia & I, II \& III & 12 & $40 \%$ & 10 & $33.3 \%$ & 0.395 \\
& & 0 & & 0 & & \\
\hline Anaemia & I, II \& III & 4 & $13.3 \%$ & 3 & $10 \%$ & 0.690 \\
& & 0 & & 0 & & \\
\hline $\begin{array}{l}\text { Thrombo- } \\
\text { cytopenia }\end{array}$ & I, II \&III & 2 & $6.7 \%$ & 1 & $3.3 \%$ & 0.5 \\
& & 0 & & 0 & & \\
\hline
\end{tabular}


Kasr-El-Aini Journal Of Clinical Oncology And Nuclear Medicine

Vol. 7 | No. 3-4 2011

Wessam Ali El-Sherief, et al.

Table 4: Non-hematological toxicities.

\begin{tabular}{|c|c|c|c|c|c|c|}
\hline \multirow[b]{2}{*}{ Parameter } & \multicolumn{2}{|c|}{ Arm I } & \multicolumn{2}{|c|}{ Arm II } & \multirow[b]{2}{*}{ Percent } & \multirow{2}{*}{$P$-value } \\
\hline & Grade & Number & Percent & Number & & \\
\hline \multirow[t]{2}{*}{ Alopecia } & I, II \& III & 6 & $20 \%$ & 2 & $6.7 \%$ & 0.12 \\
\hline & & 0 & & 0 & & \\
\hline \multirow[t]{2}{*}{ Fatigue } & I, II \& III & 9 & $30 \%$ & 9 & $30 \%$ & 0.61 \\
\hline & & 0 & & 0 & & \\
\hline \multirow[t]{2}{*}{ Nausea } & I, II \& III & 9 & $30 \%$ & 7 & $23.3 \%$ & 0.56 \\
\hline & & 0 & $0 \%$ & 0 & & \\
\hline \multirow[t]{2}{*}{ Nail changes } & I, II \& III & 0 & & 4 & $13.8 \%$ & 0.03 \\
\hline & & 0 & & 0 & & \\
\hline Fluid ret. & $>3 \mathrm{~kg}$ & 8 & $26.7 \%$ & 0 & 0 & 0.03 \\
\hline \multirow[t]{2}{*}{ Vomiting } & I, II \& III & 7 & $24.1 \%$ & 12 & $40 \%$ & 0.196 \\
\hline & & 0 & & 0 & & \\
\hline \multirow[t]{2}{*}{ Diarrhea } & I, II \& III & 0 & 0 & 4 & $13.3 \%$ & 0.038 \\
\hline & & 0 & & 0 & & \\
\hline Epiphora & Positive & 3 & $10 \%$ & 2 & $6.9 \%$ & 0.66 \\
\hline \multirow[t]{2}{*}{ Stomatitis } & I, II \& III & 7 & $23.3 \%$ & 5 & $16.7 \%$ & 0.51 \\
\hline & & 0 & & 0 & & \\
\hline \multirow[t]{2}{*}{ Neurosens } & I, II \& III & 7 & $23.3 \%$ & 1 & $3.3 \%$ & 0.02 \\
\hline & & 0 & & 0 & & \\
\hline \multirow[t]{2}{*}{ Neuromot } & I, II \& III & 3 & $10 \%$ & 0 & $0 \%$ & 0.68 \\
\hline & & 0 & & 0 & & \\
\hline
\end{tabular}

Table 5: Prognostic factors with statistical significance $(P$-value).

\begin{tabular}{lccc}
\hline Prognostic factor & Response & TTP & OAS \\
\hline Age & 0.014 & 0.034 & 0.001 \\
\hline Site of recurrence & 0.004 & 0.012 & 0.026 \\
\hline No of metastatic sites & 0.008 & 0.016 & 0.03 \\
\hline
\end{tabular}

Table 6: Prognostic factors without statistical significance ( $P$-value).

\begin{tabular}{lccc}
\hline Prognostic factor & Response & TTP & OAS \\
\hline PS & 0.794 & 0.727 & 0.317 \\
\hline MS & 0.544 & 0.118 & 0.119 \\
\hline HS & 0.452 & 0.969 & 0.668 \\
\hline
\end{tabular}

PS: Performance status, MS: Menopausal status, HS: Hormonal status.

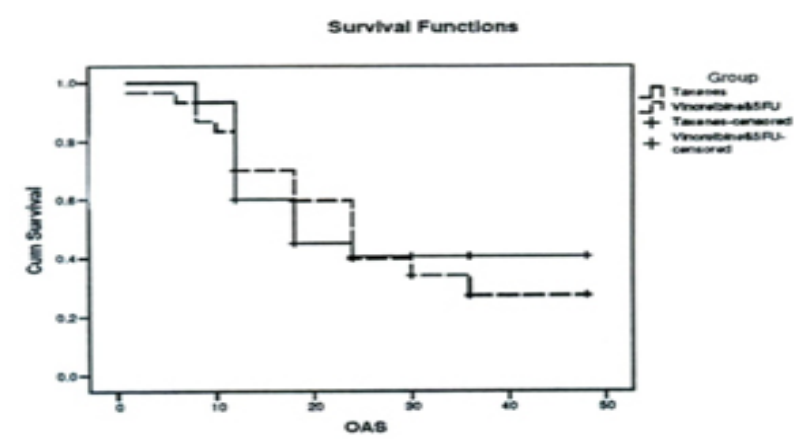

Figure 1: Overall survival.

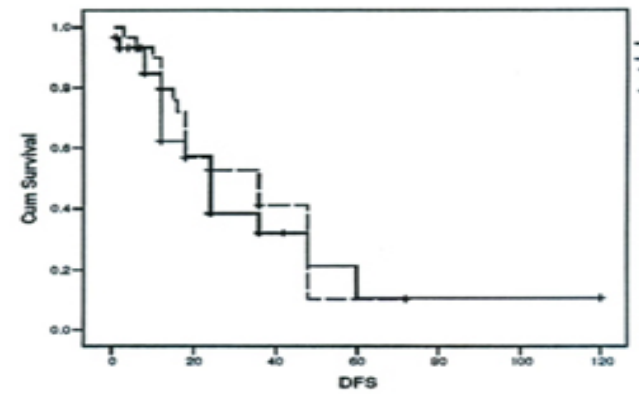

Figure 2: Progression free survival. 


\section{DISCUSSION AND RECOMMENDATION}

Breast cancer is the most common female malignancy in Egypt. It ranks the first among female malignancies.

Hamza et al. reported that breast cancer accounts for $22.6 \%$ of all cases of cancer attending the Medical Oncology outpatient clinic at the NCI, accounting for $44.8 \%$ of cases among females.

Inspite of adequate primary therapy in breast cancer, many patients with apparently localized disease harbor subclinical micrometastasis that may grow into clinically relevant macrometastases later on ${ }^{5}$.

Metastatic breast cancer patients have a median survival of 2-3 years, this occurs despite the discovery of numerous new agents that can palliate the disease and more rarely increase the overall survival ${ }^{6}$. Therefore, for the majority of patients with metastatic breast cancer "cure" is not the goal of treatment, instead, more conservative treatments are prefered to obtain maximum control of symptoms, prevent serious complications and prolong life with minimal toxicities and disruption of quality of lifes.

In the last decade the development of new cytotoxic drugs, combinations and the introduction of novel targeted agents have permitted to lengthened patient's survival and improved quality of life ${ }^{7}$.

There is no single standard chemotherapy regimen for patients with MBC. In addition to the taxanes, various agents (e.g. capecitabine, gemcitabine and vinorelbine) used alone or in combination have proved to be efficacious.

In this prospective study, we compared taxanes verus a combination of vinorelbine and fluorouracil in patients with metastatic cancer breast. Complete remission CR was $3 / 30(10.3 \%)$ in arm I (received taxanes) vs $1 / 30$ (3.4\%) in arm II (received navelbine and 5FU) with no statistically significant difference, difference in partial response and progressive disease was slightly better in the Taxane group again with no statistically significant difference.

Patients who attained partial remission PR were $13 / 30(44.8 \%)$ in arm I vs $9 / 30(31 \%)$ in arm II, while patient who developed progressive disease PD was $3 / 30$ $(10.3 \%)$ in arm I vs 6/30 (20.0\%) in arm II. The mean over all survival was 27.7 months vs 26.5 months in arm II.

Factors affecting response were age, site of recurrence and number of metastasis. All theses factors show statistically significant difference in both treatment groups, while other factors showed no statistically significant difference.

These factors are performance status PS, Menopausal Status (MS), Hormonal Status (HS) as will as Disease Free Survival (DFS). This response rate is comparaple to other studies that use the same regimen like Bonneteterre et al. ${ }^{8}$ who obtained response in 176 women with metastatic breast cancer who had failed anthracycline-based therapy The CR rate following Taxotere was $7 \%$, compared to $4.4 \%$ following 5-FU and Navelbine. The PR rate was $36 \%$ for the Taxotere group and $34.4 \%$ for the combination group. Overall survival was 16 months for the Taxotere group and 15 months for the combination group.

Also in comparison with other regimens used in $\mathrm{MBC}$ as vinorelbine and cispltin that were reported by Vassilomanlakis et al. ${ }^{9}$, who obtained response rate in 53 patients with 4 patients $(8 \%)$ in CR, 22 patients $(41 \%)$ in PR, ORR (49\%). In comparison to Gunel et al..$^{10}$ who obtaind response rate $25 \%$ in 24 patient and to RayCoquard et al. who obtaind response rate in 58 patients 2 patients (3\%) in CR, 22 patients (38\%), ORR (43\%) using the same regimen.

There is a wide range of variation between different studies using chemotherapy in MBC even between studies using the same regimen, this might be due to selection bias where these studies are mostly nonrandomized trial.

Another important factor is the use of different dose schedules among different studies, not only the scheduled dose differ from study to study but more important the relative average dose intensity differ from study to study even if they have the same dose schedule.

The number of patients needed hospitalization with blood transfusion and growth stimulating factor were 4 vs 5 for taxanes vs navelbine and 5-FU average hospital stay is four days, these adds to treatment cost.

In comparison to Bonneterre et al. ${ }^{8}$ who reported the main grade III-IV toxicities were $82 \%$ vs $67 \%$; stomatitis $5 \%$ vs $40 \%$; febrile neutopenia $13 \%$ vs $22 \%$ and infection $2 \%$ vs $7 \%$ for docetaxel vs navelbine and 5 -FU. There was one possible treatment-related death in the docetaxel arm and five with navelbine and 5-FU.

As regards cost effective outcome, the paclitaxel or docetaxel arm costs about 35.000 L.E. and the navelbine and 5-FU arm costs about 15.000 L.E on average and this reflects the economical advantage of the vinorelbine and 5 -flurouoracil. 
This economic advantage is considered as a major advantage in favor of navelbine and 5-FU in developing countries.

Clinical research in which more attention is paid to symptom control and quality of life and increased emphasis is placed on improving treatment individualization remains the only way for progress to be made in the management of MBC

\section{CONCLUSION}

In conclusion, in a situation where treatment outcome as well as toxicity profile are comparable in both treatment arms, the cost effective issue should have an important impact on treatment selection. With the growing understanding of biology of breast cancer and the advent of new techniques such as genomics and proteomics, multiple new targets for anticancer therapy are identified every year. The challenge for clinicians will be to find the most appropriate niche for the new biologic therapies in breast cancer management, the hope being that the therapies will significantly improve the quality and the length of chemotherapy induced remissions and disease stabilization.

\section{REFERENCES}

1. Orlando L, Colleoni M, Fedele P, Cusmai A, Rizzo P, D'Amico M, et al. Management of advanced breast cancer. Ann.Oncol. 2007;18(Suppl. 16):vi74-6.

2. Chung CT, Carlson RW. Goals and objectives in the management of metastatic breast cancer. Oncologist 2003;8(6):514-20.

3. Bernard Marty C, Cardoso F, Piccart MJ. Facts and controversies in systemic treatment of metastatic breast cancer. Oncologist 2004;9(6):617-32.

4. Costanza ME, Weiss RB, Henderson IC, Norton L, Berry
DA, Cirrincione C, et al. Safety and efficacy of using a single agent or a phase II agent before instituting standard combination chemotherapy in previously untreated metastatic breast cancer patients: Report of a randomized study - Cancer and Leukemia Group B 8642. J.Clin.Oncol. 1999;17(5):1397-406.

5. Colozza M, De Azambuja E, Personeni N, Lebrun F, Piccart MJ, Cardoso F. Achievements in systemic therapies in the pregenomic era in metastatic breast cancer. Oncologist 2007;12(3):253-70.

6. Bontenbal M, Creemers GJ, Braun HJ, De Boer AC, Janssen JT, Leys RB, et al. Phase II to III study comparing doxorubicin and docetaxel with fluorouracil, doxorubicin, and cyclophosphamide as first-line chemotherapy in patients with metastatic breast cancer: Results of a Dutch community setting trial for the clinical trial group of the comprehensive cancer centre. J.Clin.Oncol. 2005;23(28):7081-8.

7. Adamo V, Franchina T, Adamo B, Ferraro G, Rossello $\mathrm{R}$, Saccà MM, et al. Safety and activity of trastuzumabcontaining therapies for the treatment of metastatic breast cancer: Our long-term clinical experience (GOIM study). Ann.Oncol. 2007;18(Suppl. 6):vi11-5.

8. Bonneterre J, Roché H, Monnier A, Guastalla JP, Namer $\mathrm{M}$, Fargeot $\mathrm{P}$, et al. Docetaxel vs 5-fluorouracil plus vinorelbine in metastatic breast cancer after anthracycline therapy failure. Br.J.Cancer 2002;87(11):1210-5.

9. Vassilomanolakis M, Koumakis G, Barbounis V, Demiri M, Pateras H, Efremidis AP. Vinorelbine and cisplatin in metastatic breast cancer patients previously treated with anthracyclines. Ann.Oncol. 1000 Sep;11(9): 115-1160.

10. Gunel N, Akcali Z, Yamac D, Onuk E, Yilmaz E, Bayram $\mathrm{O}$, et al. Cisplatin plus vinorelbine as a salvage regimen in refractory breast cancer. Tumori 2000;86(4):283-5.

11. Ray Coquard I, Biron P, Bachelot T, Guastalla JP, Catimel G, Merrouche Y, et al. Vinorelbine and cisplatin (CIVIC regimen) for the treatment of metastatic breast carcinoma after failure of anthracycline- and/or paclitaxel-containing regimens. Cancer 1998;82(1):134-40. 